\title{
Analytical Solution of Two Extended Model Equations for Shallow Water Waves by Adomian's Decomposition Method
}

\author{
Mehdi Safari \\ Islamic Azad University, Aligoodarz Branch, Department of Mechanical Engineering, Aligoodarz, Iran \\ E-mail:ms_safari2005@yahoo.com \\ Received May 22, 2011; revised June 26, 2011; accepted July 5, 2011
}

\begin{abstract}
In this paper, we consider two extended model equations for shallow water waves. We use Adomian's decomposition method (ADM) to solve them. It is proved that this method is a very good tool for shallow water wave equations and the obtained solutions are shown graphically.
\end{abstract}

Keywords: Adomian’s Decomposition Method, Shallow Water Wave Equation

\section{Introduction}

Clarkson et al [1] investigated the generalized short water wave (GSWW) equation

$$
u_{t}-u_{x x t}-\alpha u u_{t}-\beta u_{x} \int^{x} u_{t} \mathrm{~d} x+u_{x}=0
$$

where $\alpha$ and $\beta$ are non-zero constants.

Ablowitz et al. [2] studied the specific case $\alpha=4$ and $\beta=2$ where Equation (1) is reduced to

$$
u_{t}-u_{x x t}-4 u u_{t}-2 u_{x} \int^{x} u_{t} \mathrm{~d} x+u_{x}=0
$$

This equation was introduced as a model equation which reduces to the $\mathrm{KdV}$ equation in the long small amplitude limit [2,3].However, Hirota et al. [3] examined the model equation for shallow water waves

$$
u_{t}-u_{x x t}-3 u u_{t}-3 u_{x} \int^{x} u_{t} \mathrm{~d} x+u_{x}=0
$$

obtained by substituting $\alpha=\beta=3$ in (1).

Equation (2) can be transformed to the bilinear forms

$$
\left[D_{x}\left(D_{t}-D_{t} D_{x}^{2}+D_{x}\right)+\frac{1}{3} D_{t}\left(D_{s}+D_{x}^{3}\right)\right] f \cdot f=0
$$

where $s$ is an auxiliary variable, and $f$ satisfies the bilinear equation

$$
D_{x}\left(D_{s}+D_{x}^{3}\right) f \cdot f=0
$$

However, Equation (3) can be transformed to the bilinear form

$$
D_{x}\left(D_{t}-D_{t} D_{x}^{2}+D_{x}\right) f \cdot f=0
$$

where the solution of the equation is

$$
u(x, t)=2(\ln f)_{x x}
$$

where $f(x, t)$ is given by the perturbation expansion

$$
f(x, t)=1+\sum_{n=1}^{\infty} \varepsilon^{n} f_{n}(x, t)
$$

where $\varepsilon$ is a bookkeeping non-small parameter, and $f_{n}(x, t), n=1,2, \cdots, n$ are unknown functions that will be determined by substituting the last equation into the bilinear form and solving the resulting equations by equating different powers of $\varepsilon$ to zero.

The customary definition of the Hirota's bilinear operators are given by

$$
\begin{aligned}
& D_{t}^{n} D_{x}^{m} a \cdot b \\
& =\left(\frac{\partial}{\partial t}-\frac{\partial}{\partial t^{\prime}}\right)^{n}\left(\frac{\partial}{\partial x}-\frac{\partial}{\partial x^{\prime}}\right)^{m} a(x, t) b\left(x^{\prime}, t^{\prime}\right) \mid x^{\prime}=x, t^{\prime}=t
\end{aligned}
$$

Some of the properties of the $D$-operators are as follows

$$
\begin{aligned}
& \frac{D_{t}^{2} f \cdot f}{f^{2}}=\iint u_{t t} \mathrm{~d} x \mathrm{~d} x, \frac{D_{t} D_{x}^{3} f \cdot f}{f^{2}}=u_{x t}+3 u \int x u_{t} \mathrm{~d} x^{\prime} \\
& \frac{D_{x}^{2} f \cdot f}{f^{2}}=u, \frac{D_{x}^{4} f \cdot f}{f^{2}}=u_{2 x}+3 u^{2}, \frac{D_{t} D_{x} f \cdot f}{f^{2}}=\ln \left(f^{2}\right)_{x t} \\
& \frac{D_{x}^{6} f \cdot f}{f^{2}}=u_{4 x}+15 u u_{2 x}+15 u^{3}
\end{aligned}
$$


where

$$
u(x, t)=2(\ln f(x, t))_{x x}
$$

Also extended model of Equation (2) is obtained by the operator $D_{x}^{4}$ to the bilinear forms (4) and (5)

$$
\left[D_{x}\left(D_{t}-D_{t} D_{x}^{2}+D_{x}+D_{x}^{3}\right)+\frac{1}{3} D_{t}\left(D_{s}+D_{x}^{3}\right)\right] f \cdot f=0
$$

where $\mathrm{s}$ is an auxiliary variable, and $f$ satisfies the bilinear equation

$$
D_{x}\left(D_{s}+D_{x}^{3}\right) f \cdot f=0
$$

Using the properties of the $\mathrm{D}$ operators given above, and differentiating with respect to $x$ we obtain the extended model for Equation (2) given by

$$
u_{t}-u_{x x t}-4 u u_{t}-2 u_{x} \int^{x} u_{t} \mathrm{~d} x+u_{x}+u_{x x x}+6 u u_{x}=0
$$

In a like manner, we extend Equation(3) by adding the operator $D_{x}^{4}$ to the bilinear forms (6) to obtain

$$
D_{x}\left(D_{t}-D_{t} D_{x}^{2}+D_{x}+D_{x}^{3}\right) f \cdot f=0
$$

Using the properties of the $\mathrm{D}$ operators given above we obtain the extended model for Equation(3) given by

$$
u_{t}-u_{x x t}-3 u u_{t}-3 u_{x} \int^{x} u_{t} \mathrm{~d} x+u_{x}+u_{x x x}+6 u u_{x}=0
$$

In this paper, we use the Adomian's decomposition method (ADM) to obtain the solution of two considered equations above for shallow water waves. Large classes of linear and nonlinear differential equations, both ordinary as well as partial, can be solved by the ADM [4-15]. A reliable modification of ADM has been done by Wazwaz [16].The decomposition method provides an effective procedure for analytical solution of a wide and general class of dynamical systems representing real physical problems [4-14].This method efficiently works for initial-value or boundary-value problems and for linear or nonlinear, ordinary or partial differential equations and even for stochastic systems. Moreover, we have the advantage of a single global method for solving ordinary or partial differential equations as well as many types of other equations.

\section{Basic idea of Adomian's Decomposition Method}

We begin with the equation

$$
L u+R(u)+F(u)=g(t)
$$

where $L$ is the operator of the highest-ordered derivatives with respect to $t$ and $R$ is the remainder of the linear op- erator. The nonlinear term is represented by $F(u)$. Thus we get

$$
L u=g(t)-R(u)-F(u)
$$

The inverse $L^{-1}$ is assumed an integral operator given by

$$
L_{t}^{-1}=\int_{0}^{t}(\cdot) \mathrm{d} t
$$

The operating with the operator $L^{-1}$ on both sides of Equation (18) we have

$$
u=f_{0}+L^{-1}(g(t)-R(u)-F(u))
$$

where $f_{0}$ is the solution of homogeneous equation

$$
L u=0
$$

involving the constants of integration. The integration constants involved in the solution of homogeneous equation (21) are to be determined by the initial or boundary condition according as the problem is initial-value problem or boundary-value problem.

The ADM assumes that the unknown function $u(x, t)$ can be expressed by an infinite series of the form

$$
u(x, t)=\sum_{n=0}^{\infty} u_{n}(x, t)
$$

and the nonlinear operator $F(u)$ can be decomposed by an infinite series of polynomials given by

$$
F(u)=\sum_{n=0}^{\infty} A_{n}
$$

where $u_{n}(x, t)$ will be determined recurrently, and $A_{n}$ are the so-called polynomials of $u_{0}, u_{1}, \cdots, u_{n}$ defined by

$$
A n=\frac{1}{n !} \frac{\mathrm{d}^{n}}{\mathrm{~d} \lambda^{n}}\left[F\left(\sum_{n=0}^{\infty} \lambda^{i} u_{i}\right)\right]_{\lambda=0}, n=0,1,2, \cdots
$$

\section{ADM Implement for First Extended Model of Shallow Water Wave Equation}

We consider the application of ADM to first extended model of shallow water wave equation. If Equation (14) is dealt with this method, it is formed as

$$
\begin{aligned}
L_{t} u & =L_{x x t} u+4 u L_{t} u+2 L_{x} u \int^{x} L_{t} u \mathrm{~d} x \\
& -L_{x} u-L_{x x x} u-6 u L_{x} u
\end{aligned}
$$

where

$$
L_{x x t}=\frac{\partial^{3}}{\partial x^{2} \partial t}, L_{x}=\frac{\partial}{\partial x}, L_{x x t}=\frac{\partial^{3}}{\partial x^{2} \partial t}, L_{x x x}=\frac{\partial^{3}}{\partial x^{3}}
$$

If the invertible operator $L_{t}^{-1}=\int_{0}^{t}(\cdot) \mathrm{d} t$ is applied to Equation (25), then 


$$
\begin{aligned}
L_{t}^{-1} L_{t} u & =L_{t}^{-1}\left(L_{x x t} u+4 u L_{t} u+2 L_{x} u \int^{x} L_{t} u \mathrm{~d} x\right. \\
& \left.-L_{x} u-L_{x x x} u-6 u L_{x} u\right)
\end{aligned}
$$

is obtained. By this

$$
\begin{aligned}
u(x, t) & =u(x, 0)+L_{t}^{-1}\left(L_{x x t} u+4 u L_{t} u+2 L_{x} u \int^{x} L_{t} u \mathrm{~d} x\right. \\
& \left.-L_{x} u-L_{x x x} u-6 u L_{x} u\right)
\end{aligned}
$$

is found. Here the main point is that the solution of the decomposition method is in the form of

$$
u(x, t)=\sum_{n=0}^{\infty} u_{n}(x, t)
$$

Substituting from Equation (29) in Equation(28), we find

$$
\begin{aligned}
\sum_{n=0}^{\infty} u_{n}(x, t) & =u(x, 0)+L_{t}^{-1}\left(L_{x x t}\left(\sum_{n=0}^{\infty} u_{n}(x, t)\right)\right. \\
& +4\left(\sum_{n=0}^{\infty} u_{n}(x, t)\right) L_{t}\left(\sum_{n=0}^{\infty} u_{n}(x, t)\right) \\
& +2 L_{x}\left(\sum_{n=0}^{\infty} u_{n}(x, t)\right) \int^{x} L_{t}\left(\sum_{n=0}^{\infty} u_{n}(x, t)\right) \mathrm{d} x \\
& -L_{x}\left(\sum_{n=0}^{\infty} u_{n}(x, t)\right)-L_{x x x}\left(\sum_{n=0}^{\infty} u_{n}(x, t)\right) \\
& \left.-6\left(\sum_{n=0}^{\infty} u_{n}(x, t)\right) L_{x}\left(\sum_{n=0}^{\infty} u_{n}(x, t)\right)\right)
\end{aligned}
$$

is found.

According to Equation (19) approximate solution can be obtained as follows:

$$
\begin{aligned}
& u_{0}(x, t)=\frac{(c-1) \operatorname{sech}^{2}\left(\frac{1}{2} \sqrt{\frac{c-1}{c+1}} x\right)}{2 c+2} \\
& u_{1}(x, t)=\frac{\sinh \left(\frac{1}{2} \sqrt{\frac{c-1}{c+1}} x\right) \sqrt{\frac{c-1}{c+1}} t c(c-1)}{\cosh ^{3}\left(\frac{1}{2} \sqrt{\frac{c-1}{c+1}} x\right)(c+1)^{2}} \\
& u_{2}(x, t)=\int_{0}^{t}\left(L_{x x t} u_{1}+4 u_{1} L_{t} u_{1}+2 L_{x} u_{1} \int^{x} L_{t} u_{1} \mathrm{~d} x\right. \\
& \left.-L_{x} u_{1}-L_{x x x} u_{1}-6 u_{1} L_{x} u_{1}\right) \mathrm{d} t
\end{aligned}
$$

Thus the approximate solution for first extended model of shallow water wave equation is obtained as

$$
u(x, t)=u_{0}(x, t)+u_{1}(x, t)+u_{2}(x, t)
$$

The terms $u_{0}(x, t), u_{1}(x, t), u_{2}(x, t)$ in Equation (34), obtained from Eqs. (31), (32), (33).

\section{ADM Implement for Second Extended Model of Shallow Water Wave Equation}

Here we consider the application of ADM to second extended model of shallow water wave equation. If Equation (16) is dealt with this method, it is formed as

$$
L_{t} u=L_{x x t} u+3 u L_{t} u+3 L_{x} u \int^{x} L_{t} u \mathrm{~d} x-L_{x} u-L_{x x x} u-6 u L_{x} u
$$

where

$$
L_{t}=\frac{\partial}{\partial t}, L_{x}=\frac{\partial}{\partial x}, L_{x x t}=\frac{\partial^{3}}{\partial x^{2} \partial t}, L_{x x x}=\frac{\partial^{3}}{\partial x^{3}}
$$

If the invertible operator $L_{t}^{-1}=\int_{0}^{t}(\cdot) \mathrm{d} t$ is applied to Equation (35), then

$$
\begin{aligned}
L_{t}^{-1} L_{t} u & =L_{t}^{-1}\left(L_{x x t} u+3 u L_{t} u+3 L_{x} u \int^{x} L_{t} u \mathrm{~d} x\right. \\
& \left.-L_{x} u-L_{x x x} u-6 u L_{x} u\right)
\end{aligned}
$$

is obtained. By this

$$
\begin{aligned}
u(x, t) & =u(x, 0)+L_{t}^{-1}\left(L_{x x t} u+3 u L_{t} u+3 L_{x} u \int^{x} L_{t} u \mathrm{~d} x\right. \\
& \left.-L_{x} u-L_{x x x} u-6 u L_{x} u\right)
\end{aligned}
$$

is found. Here the main point is that the solution of the decomposition method is in the form of

$$
u(x, t)=\sum_{n=0}^{\infty} u_{n}(x, t)
$$

Substituting from Equation (39) in Equation (38), we find

$$
\begin{aligned}
\sum_{n=0}^{\infty} u_{n}(x, t) & =u(x, 0)+L_{t}^{-1}\left(L_{x x t}\left(\sum_{n=0}^{\infty} u_{n}(x, t)\right)\right. \\
& +3\left(\sum_{n=0}^{\infty} u_{n}(x, t)\right) L_{t}\left(\sum_{n=0}^{\infty} u_{n}(x, t)\right) \\
& +3 L_{x}\left(\sum_{n=0}^{\infty} u_{n}(x, t)\right) \int^{x} L_{t}\left(\sum_{n=0}^{\infty} u_{n}(x, t)\right) \mathrm{d} x \\
& -L_{x}\left(\sum_{n=0}^{\infty} u_{n}(x, t)\right)-L_{x x x}\left(\sum_{n=0}^{\infty} u_{n}(x, t)\right) \\
& \left.-6\left(\sum_{n=0}^{\infty} u_{n}(x, t)\right) L_{x}\left(\sum_{n=0}^{\infty} u_{n}(x, t)\right)\right)
\end{aligned}
$$




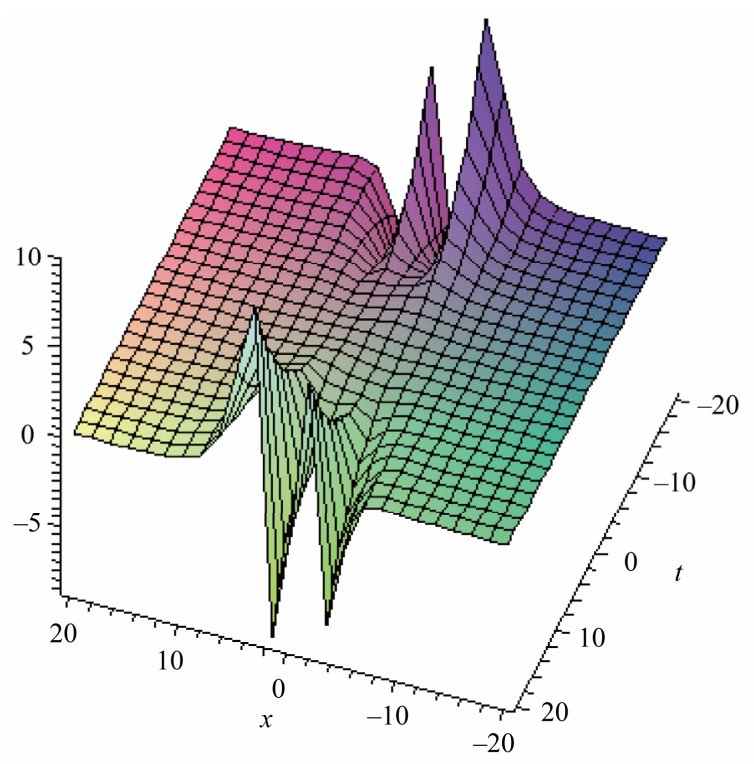

Figure 1. For the first extended model of shallow water wave equation with the first initial condition (31) of Equation (14), ADM result for $u(x, t)$, when $c=2$.

is found.

According to Equation (19) approximate solution can be obtained as follows:

$$
\begin{aligned}
& u_{0}(x, t)=\frac{(c-1) \operatorname{sech}^{2}\left(\frac{1}{2} \sqrt{\frac{c-1}{c+1}} x\right)}{2 c+2} \\
& u_{1}(x, t)=\frac{\sinh \left(\frac{1}{2} \sqrt{\frac{c-1}{c+1}} x\right) \sqrt{\frac{c-1}{c+1}} t c(c-1)}{\cosh ^{3}\left(\frac{1}{2} \sqrt{\frac{c-1}{c+1}} x\right)(c+1)^{2}} \\
& u_{2}(x, t)=\int_{0}^{t}\left(L_{x x t} u_{1}+3 u_{1} L_{t} u_{1}+3 L_{x} u_{1} \int^{x} L_{t} u_{1} \mathrm{~d} x\right. \\
& \left.-L_{x} u_{1}-L_{x x x} u_{1}-6 u_{1} L_{x} u_{1}\right) \mathrm{d} t
\end{aligned}
$$

Thus the approximate solution for second extended model of shallow water wave equation is obtained as

$$
u(x, t)=u_{0}(x, t)+u_{1}(x, t)+u_{2}(x, t)
$$

The terms $u_{0}(x, t), u_{1}(x, t), u_{2}(x, t)$ in Equation (44), obtained from Equations (41), (42), (43).

\section{Conclusions}

In this paper, Adomian's decomposition method have been successfully applied to find the solution of two extended model equations for shallow water. The obtained results were showed graphically it is proved that Ado-

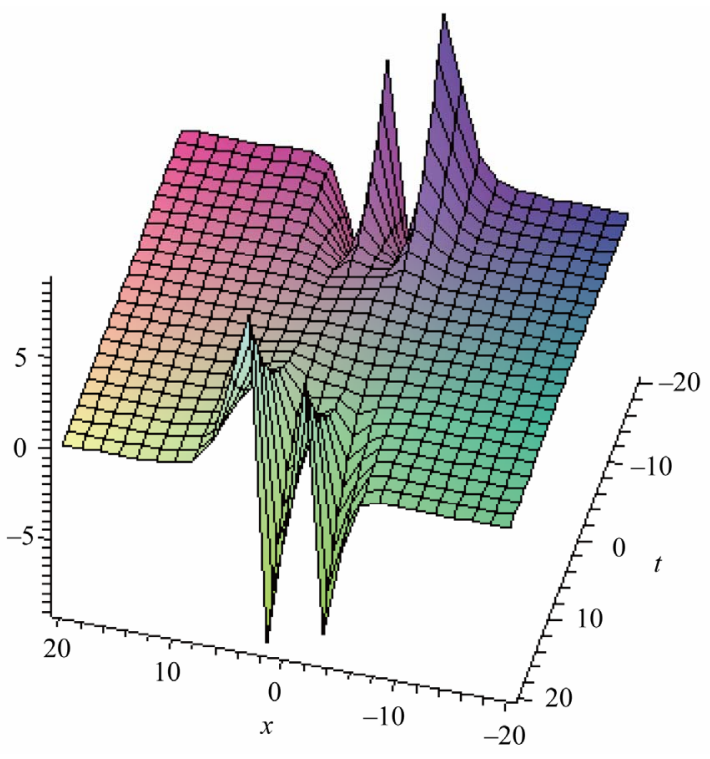

Figure 2. For the second extended model of shallow waterwave equation with the first initial condition (31) of Equation (16), ADM result for $u(x, t)$, when $c=2$.

mian's decomposition method is a powerful method for solving these equations. In our work; we used the Maple Package to calculate the functions obtained from the Adomian's decomposition method.

\section{References}

[1] P. A. Clarkson and E. L. Mansfield, "On a Shallow Water Wave Equation,” Nonlinearity, Vol. 7, No. 3, 1994, pp. 975-1000. doi:10.1088/0951-7715/7/3/012

[2] M. J. Ablowitz, D. J. Kaup, A. C. Newell and H. Segur, "The Inverse Scattering Transform-Fourier Analysis for Nonlinear Problems," Studies in Applied Mathematics, Vol. 53, 1974, pp. 249-315.

[3] R. Hirota and J. Satsuma, "Soliton Solutions of Model Equations for Shallow Water Waves," Journal of the Physical Society of Japan, Vol. 40, No. 2, 1976, pp. 611612. doi:10.1143/JPSJ.40.611

[4] G. Adomian, "An Analytical Solution of the Stochastic Navier-Stokes System,” Foundations of Physics, Vol. 21, No. 7, 1991, pp. 831-843. doi:10.1007/BF00733348

[5] G. Adomian and R. Rach, "Linear and Nonlinear Schrödinger Equations,” Foundations of Physics, Vol. 21, No. 8, 1991, pp. 983-991. doi:10.1007/BF00733220

[6] G. Adomian, "Solution of Physical Problems by Decomposition," Computers \& Mathematics with Applications, Vol. 27, No. 9/10, 1994, pp. 145-154. doi:10.1016/0898-1221(94)90132-5

[7] G. Adomian, "Solutions of Nonlinear PDE," Applied Mathematics Letters, Vol. 11, No. 3, 1998, pp. 121-123. doi:10.1016/S0893-9659(98)00043-3

[8] K. Abbaoui and Y. Cherruault, “The Decomposition met- 
hod Applied to the Cauchy Problem," Kybernetes, Vol. 28, No. 1, 1999, pp. 103-108. doi:10.1108/03684929910253261

[9] D. Kaya and A. Yokus, “A Numerical Comparison of Partial Solutions in the Decomposition Method for Linear and Nonlinear Partial Differential Equations," Mathematics and Computers in Simulation, Vol. 60, No. 6, 2002, pp. 507-512. doi:10.1016/S0378-4754(01)00438-4

[10] A. M. Wazwaz, "Partial Differential Equations: Methods and Applications,” Balkema, Rottesdam, 2002.

[11] A. M. Wazwaz, "The Extended Tanh Method for New Solitons Solutions for Many Forms of the Fifth-Order KdV Equations," Applied Mathematics and Computation, Vol. 184, No. 2, 2007, pp. 1002-1014. doi:10.1016/j.amc.2006.07.002

[12] A. M. Wazwaz, "The Tanh-Coth Method for Solitons and Kink Solutions for Nonlinear Parabolic Equations," Applied Mathematics and Computation, Vol. 188, No. 2, 2007, pp. 1467-1475. doi:10.1016/j.amc.2006.11.013

[13] D. D. Ganji, E. M. M. Sadeghi and M. Safari, “Application of He's Variational Iteration Method and Adomian's
Decom-Position Method to Prochhammer Chree Equation,” International Journal of Modern Physics B, Vol. 23, No. 3, 2009, pp. 435-446. doi:10.1142/S0217979209049656

[14] M. Safari, D. D. Ganji and M. Moslemi, “Application of He's Variational Iteration Method and Adomian's Decomposition Method to the Fractional KdV-BurgersKuramoto Equation," Computers and Mathematics with Applications, Vol. 58, No. 11-12, 2009, pp. 2091-2097. doi:10.1016/j.camwa.2009.03.043

[15] D. D. Ganji, M. Safari and R. Ghayor, "Application of He's Variational Iteration Method and Adomian's Decomposition Method to Sawada-Kotera-Ito Seventh-order Equation,” Numerical Methods for Partial Differential Equations, Vol. 27, No. 4, 2011, pp. 887-897. doi:10.1002/num.20559

[16] A. M. Wazwaz, "A Reliable Modification of Adomian Decomposition Method,” Applied Mathematics and Computation, Vol. 102, No. 1, 1999, pp. 77-86. doi:10.1016/S0096-3003(98)10024-3 